\title{
Implicaciones geopolíticas y económicas del Brexit en la Unión Europea
}

\author{
Maricel Linares Giraldo* \\ William Rodrigo Avendaño Castro ${ }^{* *}$ \\ Johanna Milena Mogrovejo Andrade ${ }^{* * *}$
}

Fecha de recepción: 1 de abril de 2020

Fecha de aceptación: 3 de junio de 2020

Resumen: El objetivo de este artículo de investigación es analizar las implicaciones económicas y geopolíticas del Brexit para la Unión Europea y Gran Bretaña. Corresponde a una investigación cualitativa con alcance descriptivo, enmarcada en el paradigma interpretativo. Se consultaron fuentes documentales para el logro de los objetivos, y la información recolectada fue sometida a un proceso de codificación y categorización a través del software Atlas Ti. Los resultados del análisis permiten exponer los hallazgos en dos apartados: 1) las causas y el proceso del Brexit, haciendo especial alusión a las razones políticas y a los resultados del voto del 2016; 2) los impactos del Brexit de acuerdo con el análisis de la literatura, los cuales se codifican y categorizan para una mejor comprensión del fenómeno. Aunque existe una importante literatura sobre los múltiples y variados efectos del Brexit, solo en los próximos años se podrán reconocer las implicaciones de este fenómeno para los grupos de interés.

Palabras clave: Brexit, Gran Bretaña, Unión Europea, integración económica, política comercial; comercio internacional.

Clasificación JEL: F02, F13, F15, F51, F53.

Cómo citar este artículo/ To reference this article / Comment citer cet article / Para citar este artigo:

Linares Giraldo, M., Avendaño Castro, W., \& Mogrovejo Andrade, J. (2020). Implicaciones geopolíticas y económicas del Brexit en la Unión Europea. Apuntes Del Cenes, 39(70). Págs. 17 - 41

https://doi.org/10.19053/01203053.v39.n70.2020.10824

* Magíster en Gestión Humana, Universidad de Antioquia, Medellín, Colombia. Docente investigadora, Universidad Francisco de Paula Santander, Cúcuta, Colombia. Correo electrónico: maricellg@ufps.edu.co (iD http://orcid.org/0000-0002-4936-1822

** Doctor en Ciencias Sociales y Humanas, Pontificia Universidad Javeriana, Bogotá, Colombia. Docente investigador, Universidad Francisco de Paula Santander, Cúcuta, Colombia. Correo electrónico: williamavendano@ufps.edu.co (i) https://orcid.org/0000-0002-7510-8222

*** Doctor en Estudios Políticos, Universidad Externado, Bogotá, Colombia. Docente investigadora, Universidad Francisco de Paula Santander, Cúcuta, Colombia. Correo electrónico: johannamogrovejo@ufps.edu.co (D) http://orcid.org/0000-0002-9694-3382 


\title{
Geopolitical and Economic Implications of Brexit in the European Union
}

\begin{abstract}
The objective of this research article is to analyze the economic and geopolitical implications of Brexit for the European Union and Great Britain. It corresponds to qualitative research with descriptive scope, framed in the interpretive paradigm. Documentary sources were consulted to achieve the objectives, and the information collected was submitted to a coding and categorization process through the Atlas Ti software. The results of the analysis allow us to present the findings in two sections: 1) the causes and process of Brexit, making special reference to the political reasons and the results of the 2016 vote; 2) the impacts of Brexit according to the analysis of the literature, which are codified and categorized for a better understanding of the phenomenon. Although there is an important literature on the many and varied effects of Brexit, only in the next few years can the implications of this phenomenon be recognized for interest groups.
\end{abstract}

Keywords: Brexit; United Kingdom; European Union; Economic Integration; Commercial Policy; International Tradecomplaint. 


\section{INTRODUCCIÓN}

Desde el año 2016, el término "Brexit" se convirtió en tendencia, y como fenómeno de amplio interés, ha sido objeto de variados análisis y reflexiones en los ámbitos académico, social, político y económico. Una de sus principales características es que genera una amplia incertidumbre sobre sus efectos e implicaciones en diferentes niveles (Belke et al., 2018; Steinberg, 2019; Graziano et al., 2018; García \& García, 2017). El mismo 24 de junio del 2016, la libra esterlina se desplomaba frente al dólar y billones de dólares eran eliminados de las acciones en el mundo:

Hubo una sensación de conmoción e incredulidad en las primeras horas de la mañana del 24 de junio de 2016, tanto en Gran Bretaña como en las capitales europeas, cuando quedó claro que una pequeña mayoría (51,9 por ciento) de los votantes británicos habían emitido su voto a favor de irse de la Unión Europea (UE). Los mercados reaccio- naron rápidamente a la votación del Brexit: la libra esterlina se desplomó a un mínimo de 31-años frente al dólar y más de 2 billones de dólares se borraron de las acciones a nivel mundial. (Hobolt, 2016, p. 1259)

La expresión Brexit configura una abreviatura para las palabras Britain que significa Gran Bretaña y exit que traduce salida. Se hace referencia al retiro de Gran Bretaña ${ }^{1}$ del bloque denominado la Unión Europea y del cual hace parte desde enero de 1973. A partir de un referendo celebrado en junio de 2016, los ciudadanos de Gran Bretaña y su Gobierno decidieron abandonar la Unión Europea, hecho que se debió quedar en pie en marzo de 2019, pero que se perfeccionó hasta enero del 2020. Claro está, no es la primera vez que algún país decide abandonar la Unión Europea, con fundamento en el artículo 49A del Tratado de Lisboa suscrito el 13 de diciembre de 2007 por los miembros de la Unión (Riordan, 2016; García \&

1 Resulta preciso señalar las diferencias entre los términos Reino Unido y Gran Bretaña. En el primer caso, el concepto engloba cuatro naciones: Inglaterra, Escocia, Irlanda del Norte y Gales. En el segundo caso, Gran Bretaña integra a Inglaterra, Escocia y Gales. De allí que se cree una especial incertidumbre sobre el destino de Irlanda del Norte. 
García, 2017). Sin embargo, el caso de Gran Bretaña ha levantado una gran agitación política.

El Tratado de Lisboa entró en vigencia a finales del 2009 modificando el Tratado de la Unión Europea del 7 de febrero de 1992, y conforme al artículo 50 de este último, "todo Estado miembro [puede] retirarse de la Unión de conformidad con sus propios requisitos constitucionales", es decir, a través del cumplimiento de exigencias establecidas dentro de sus propias cartas políticas para la denuncia de los tratados internacionales suscritos. Así mismo, esta norma establece que para retirarse de la Unión se exige: 1. Notificación al Consejo Europeo de la intención de retiro, 2. Negociar con la Unión y celebrar un nuevo acuerdo relacionado con la modalidad de retirada, y 3. La aprobación del Parlamento Europeo del nuevo acuerdo.

Pese a lo anterior, las negociaciones para la salida de Gran Bretaña de la Unión Europea y un posible periodo de transición aún no se han logrado definir, y existe la posibilidad de que el Brexit no llegue a perfeccionarse. En todo caso, las implicaciones geopolíticas y económicas de la salida de Gran Bretaña de la Unión Europea han sido ampliamente debatidas dentro de la literatura, mostrando los diversos escenarios futuros y los principales temas de interés en materia de la negociación para el retiro de Gran Bretaña del bloque. Para la mayoría de los expertos, el Brexit representa un hecho con impor- tantes efectos e implicaciones para toda la Unión Europea, incluso, otros países, empresas y stakeholders, los cuales mantienen estrechas relaciones con la Unión Europea, por ejemplo, los países del África subsahariana (Silveiro, 2017), la industria española (Garza \& Amigo, 2019), los trabajadores de la Unión que se movilizan de manera constante (Pérez, 2016), Irlanda del Norte y su estatuto jurídico-político (Compains, 2017), entre otros.

La Unión Europea es un ejemplo destacado de integración regional en el mundo. Un proceso efectivo de cooperación e integración entre los Estados trae importantes y significativas consecuencias para cada uno de los actores involucrados. Potencialidades y limitaciones son observables en los casos de integración regional, pues, por un lado, se puede gozar de una mayor soberanía y emplear instrumentos para la regulación de diferentes aspectos en el ámbito interno, por ejemplo, el control de las fronteras, decidir sobre políticas económicas, sociales y territoriales, y en general, decidir en el destino de los ciudadanos; pero en un escenario de integración se reducen estas facultades, las instituciones regionales que emergen empiezan a desplazar la competencia de los Estados y generan otros beneficios para los actores (Caporaso, 2018; Laursen, 2016). Por ello, en un escenario de profunda integración, una situación como la salida de Gran Bretaña de la Unión Europea trae consigo importantes implicaciones y resultados. 
Entonces, comprender las implicaciones de un fenómeno como el Brexit no puede darse al margen del constructo 'integración regional'. Dosenrode (2016) escribe que la integración regional es entendida dentro de la literatura bien como proceso o bien como estado, pero en todo caso se materializa en la combinación de las partes de un todo. En un proceso de integración regional se transfiere poder de decisión, obligaciones, lealtades y expectativas para la creación de nuevas reglas e instituciones compartidas:

A pesar de varios intentos de definir la integración regional, no existe una definición común, lo que dificulta comparar lo que a veces se describe como 'integración asiática' o 'integración latinoamericana' con lo que ocurre en África o Europa. [...]

En otras palabras, la integración regional es un proceso de transferencia de lealtad, expectativas y poder de decisión política $\mathrm{o}$, con un concepto obsoleto, pero aún popular, la 'soberanía' a un nuevo centro. (Dosenrode, 2016, pp. 3, 4)

Los aportes desde el ámbito académico muestran que no hay consenso sobre el concepto, y ello se debe a que cada caso de integración en particular manifiesta características, elementos y dinámicas diferentes.

Si la integración regional trae importantes resultados y efectos para los actores, igualmente la salida de una de las partes genera consecuencias para todos los grupos de interés. El caso de Reino Unido frente a la Unión Europea es una de esas situaciones que deben ser objeto de reflexión y estudio para comprender la naturaleza de los procesos de integración regional y las posibles implicaciones de la desintegración frente al bloque $y$ otros actores de interés.

El objetivo de este artículo de investigación es analizar de manera crítica las implicaciones económicas y geopolíticas del Brexit para el continente europeo. Para lo anterior, se ha adelantado el análisis y la sistematización de fuentes secundarias en las que se exploran diversos tópicos y temas asociados con el Brexit y la Unión Europea. El documento se encuentra organizado en tres apartados. En una primera sección, se describen los aspectos metodológicos del estudio. Luego se aborda el fenómeno del Brexit desde una perspectiva descriptiva de la evolución, el proceso que se adelantó y las opciones para Reino Unido. En la tercera sección se examinan las implicaciones del Brexit categorizadas en dos tipos: económicas y geopolíticas. El desarrollo de esta sección se adelantó a partir de un análisis pormenorizado de estudios e investigaciones publicados desde el 2016 hasta el primer trimestre del 2020, la mayoría de estos publicados en fuentes internacionales. Por último, se presentan las conclusiones del estudio. 


\section{METODOLOGÍA}

Corresponde a un artículo de investigación. Considerando la naturaleza del objetivo y el tratamiento de los datos, esta investigación es de corte cualitativo y se inscribe en el paradigma interpretativo, porque busca ahondar en los significados de lo que se expresa de manera verbal y no verbal, y esto incluye lo que se escribe en los documentos. En la investigación cualitativa no se emplea la estadística descriptiva o inferencial como sucede en los estudios cuantitativos, sino que se emplean otros diseños que permiten ahondar en las fuentes de información y la captura de los datos (Hernández et al., 2014; Bonilla \& Rodríguez, 2005).

De igual manera, considerando el nivel o alcance de investigación, el presente estudio es descriptivo y cuenta con un diseño documental que sigue un proceso de codificación para el análisis detallado de los datos. Los textos escritos o documentos son instrumentos o artefactos que se encuentran llenos de significados, porque parten de las prácticas y comprensiones de individuos o grupos, y estos significados sirven de fuente en la construcción del saber epistémico (Mogalakwe, 2006). En esta investigación se han rastreado documentos de investigación publicados entre el 2016 y el primer trimestre del 2020, tanto en español como en inglés.
Identificadas y recuperadas las fuentes documentales, se llevó a cabo una exploración detallada del contenido. De allí se extrajeron unidades de texto que fueron sometidas a un proceso de codificación y categorización en una matriz de Excel: codificación abierta, codificación axial y categorías selectivas (Strauss \& Corbin, 2004), las cuales fueron introducidas al software Atlas Ti. El procesamiento de la información permitió construir una perspectiva global sobre las posibles implicaciones geopolíticas y económicas del Brexit hacia el futuro.

\section{EL FENÓMENO DEL BREXIT: CAUSAS Y EVOLUCIÓN}

Para analizar las razones que motivaron el Brexit, se deben considerar dos escenarios. Primero, aquellos elementos que incitaron al Gobierno del Reino Unido a considerar la oportunidad de activar el artículo 50 del Tratado de la Unión Europea para el retiro del Estado del bloque, y segundo, los elementos que pueden justificar los resultados de la votación del referendo en el 2016, y en el que un poco más del $51 \%$ de los votantes manifestó su voluntad de abandonar la Unión Europea.

Los orígenes no pueden ser ubicados en una sola dimensión, pues el Brexit es producto de diferentes causas que terminan por servir de base en dos clases de decisiones: una política, en cabeza del Gobierno, y otra civil, basada en un mecanismo de participación ciudadana 
conforme al voto de opinión de la sociedad civil con capacidad para votar. En esta última situación, se indica que la votación sobre el Brexit es un claro ejemplo de la paradoja de Condorcet en el sentido de que los votantes contravienen sus propios intereses (Paniagua, 2018; Lanchester, 2016).

Muchos destacan que desde el mismo momento en que ingresó el Reino Unido a la Unión Europea, su adhesión ya se encontraba viciada y su integración resultaba débil:

Los británicos nunca se han sentido del todo involucrados en Europa y ven a la UE desde un ángulo puramente práctico con respecto a las ventajas materiales que les proporciona. Ni los políticos ni los medios de comunicación han contribuido a que ese sentimiento se moviese más cerca, psicológicamente, 'a Europa'. (Álvarez, 2016, p. 29)

Por ejemplo, se describe que en la narrativa de la política británica siempre se ha profesado el "excepcionalismo británico" (Daddow, 2015; Álvarez, 2016), lo cual lleva a exaltar la nación del Reino Unido/Gran Bretaña sobre otros actores, y a fortalecer prejuicios de los mismos ciudadanos frente a sus pares en Europa: "Por lo tanto, podemos entender por qué Gran Bretaña se ha acercado a la puerta de salida de la UE porque los líderes británicos se han basado constantemente en narrativas de 'forasteros' como marco organizativo para sus discursos de política europea" (Daddow, 2015, p. 71). De la misma forma, la imagen histórica permite señalar que el Reino Unido siempre ha tenido una postura directa o indirecta, contraria a la integración:

Reino Unido se había instalado, desde la época de Margaret Thatcher, en una retórica contraria a la integración, no solo con la Política Exterior y su más reciente creación del Servicio Europeo de Acción Exterior, también en otros ámbitos, como Schengen, el Euro, la Carta Europea de Derechos Fundamentales, muestras, todas ellas, de un país poco entusiasmado con la construcción europea y, por ende, con la integración. (Guinea, 2018, p. 305)

\section{Hacia las razones políticas del Brexit: ¿por qué optar por la desintegración?}

Desde el primer escenario, se tiene que antes de la celebración del referendo, las declaraciones de los patrocinadores del Brexit se enfocaban en argumentos como aumentar el control sobre muchos asuntos que habían quedado limitados por pertenecer a la Unión Europea o abandonar de manera definitiva una organización extremadamente burocrática. El entonces primer ministro, David Cameron, prometió en épocas de elecciones y campaña del 2015, que realizaría un referendo con el objetivo de calmar los ánimos del Partido Conservador y decidir la permanencia del Estado dentro de la Unión Europea. 
Los partidarios de la salida del Reino Unido del bloque sostienen que la Unión Europea ha limitado y frenado la regulación de asuntos migratorios, laborales, ambientales, consumo, fiscales, entre otros.

Pero, como lo describen Álvarez (2016) y Daddow (2015), o bien como se menciona en algunos estudios sobre percepciones de los ciudadanos europeos frente a la Unión Europea -eurobarómetro- (Debomy, 2012; Signorelli, 2012), existe un discurso de exaltación del Reino Unido que recorre tanto el sector político como la misma sociedad civil. Mientras que los ciudadanos de otros países muestran entender la necesidad de una Europa unida y fuerte, en Gran Bretaña el discurso cambia y se inclina hacia la defensa de lo interno y el rechazo de los otros actores.

De esta manera, se entiende que hay una cierta postura nacionalista -un componente ideológico- al querer controlar y decidir sobre variados temas y asuntos que han sido limitados por un tratado como el que cobija a los Estados que integran la Unión Europea. Y en efecto, se debe considerar que en las relaciones interestatales se puede llegar a diferentes niveles de interacción dependiendo de los objetivos y propósitos perseguidos. Al menos tres grados de interacción se pueden distinguir entre los Estados: concertación, cooperación e integración (Franco \& Robles, 1995; Tokatlian, 1994). La Unión Europea es un caso exclusivo de integración y no de concertación o cooperación meramente. La integración corresponde a un proceso complejo en el que las dinámicas en diversas dimensiones de la vida se ven afectadas:

[...] por integración debe entenderse un proceso más amplio, complejo y profundo entre dos o más naciones que implican una vinculación e interdependencia social, política, económica, cultural, científica, diplomática o incluso militar de enormes proporciones, y con un papel dinámico y protagónico de variados agentes de las sociedades involucradas. En este caso la dimensión de integración es lo suficientemente compleja como para afectar a la sociedad civil y comprometer la viabilidad política y económica de una nación. (Franco \& Robles, 1995, p. 18)

La construcción de puentes en zonas de frontera, los acuerdos para reducir aranceles, un mejor tratamiento en impuestos, acuerdos de naturaleza educativa para la homologación de títulos, el permitir el paso de en las fronteras de las poblaciones de un lado y otro, etcétera, son meras prácticas de concertación y cooperación.

Los autores citados, Franco y Robles (1995), indican que la concertación se da en el terreno estatal y en el campo diplomático, y tiende al desarrollo de tareas conjuntas para la satisfacción de objetivos a corto plazo, v.g., la delimitación de áreas submarinas o la formación de comisiones bilaterales en temas específicos. Por otro lado, en 
la cooperación media un proceso de negociación y se generan algunas obligaciones reciprocas. Por lo general, los objetivos o propósitos en el terreno de la cooperación son más amplios y pueden, según el grado de interacción, dar paso a la integración.

Entonces, la integración involucra lo económico y lo científico, también lo diplomático y militar, lo político y social, e incluso, lo cultural. De esta manera, no solo el Estado se ve afectado, sino toda una multiplicidad de actores como los no estatales, las empresas y la misma sociedad civil. Esto se debe a los fuertes compromisos que se generan y a la disminución de la voluntad para decidir sobre determinados asuntos, porque se cede parte de la soberanía y capacidad de gobernarse a cambio de los beneficios de la integración.

Ánima (2017) indica que en los últimos años la Unión Europea ha venido atravesando diversos problemas y dificultades en materia de integración que han llevado a cuestionar su viabilidad. Entre los aspectos más importantes se encuentra el grado de vulnerabilidad frente a las crisis económicas globales, lo que se opone al objetivo de fortalecimiento económico que sirvió de base para la creación de la Unión Europea. De la misma forma, la autora sostiene que los indicadores económicos globales de los países que integran la Unión se ven sometidos a fluctuaciones desde la recesión del periodo 2008-2009, y esto ha llevado a políticas de respuesta que aumentan el déficit en el gasto público y el endeudamiento, y que reducen el gasto comprometiendo el bienestar de los ciudadanos.

Las limitaciones y problemas internos, es decir, en cada uno de los países, son numerosos, y esta situación no les permite actuar en bloque y mantener la integración que caracteriza a la Unión. Por ello, es necesario que sean resueltos los problemas internos a fin de mejorar las condiciones que conduzcan a una actuación eficiente en bloque, pues, de lo contrario, se acrecientan los conflictos socioeconómicos impidiendo el proceso de integración (Ánima, 2017). Desde esta perspectiva, se observa que la integración como modalidad de interacción entre Estados no termina o finaliza con la firma de tratados o acuerdos bilaterales o multilaterales, sino que configura un proceso de constante y permanente construcción en el que resulta fundamental una visión de abajo-arriba $y$ viceversa. En este punto resulta fundamental considerar las posturas y las voces de los ciudadanos (Debomy, 2012).

Dicho de otra manera, la integración exige un trabajo permanente $\mathrm{y}$, de ser necesario, incluso la reestructuración de los compromisos, las reglas y las normas, porque de lo contrario, se hace imposible atender la demanda de problemas, limitaciones y dificultades que se presentan en la diversas dimensiones -política, social, económica, ambiental, seguridad, tecnológica, etcétera-. Cada Estado debe tener un cierto control 
sobre sus asuntos y brindar respuestas efectivas y oportunas a sus problemas con el objetivo de poder ofrecer un perfil competitivo para actuar en conjunto, pues a contrario sensu, sus limitaciones y problemas pueden afectar de manera global a los demás países.

Al parecer, se trata de verdaderas paradojas de la integración. Desde la perspectiva conceptual, se reconoce en la figura de la integración un mecanismo para promover cambios, satisfacer necesidades, generar el apoyo mutuo, maximizar el alcance de los recursos públicos y aumentar el bienestar de las comunidades. Sin embargo, las experiencias de integración, incluyendo el caso de la Unión Europea, muestran que si bien la ventaja comparativa es la razón por la cual se promueve este tipo de mecanismos, se genera menor satisfacción para las democracias, aumento de desigualdad y pobreza pese al aumento del intercambio comercial, y el desarrollo de pocas acciones en concreto para la integración (Altmann, 2011; Debomy, 2012).

Además del excepcionalismo británico ya descrito en páginas anteriores, otro factor causal doméstico de tipo estructural es el "euroescepticismo" (Álvarez, 2016; Hobolt, 2016). Este concepto surgió en el Reino Unido a mediados de la década de los 80 del siglo XX y se materializa en posturas contrarias a todas aquellas políticas que facilitan la integración, por ejemplo, las propuestas de libertad migratoria o las políticas para la unificación de la moneda. Como en el caso del rechazo al euro como moneda común, que se convirtió en un asunto generalizado en el Reino Unido (Álvarez, 2012; Álvarez, 2016; Guinea, 2018). Es posible que durante décadas se alimentara desde el ámbito público y político un imaginario / ideología de oposición a la idea de la Europa integrada y, por ello, la conducta ambivalente de los gobiernos respecto de una integración efectiva y plena con Europa:

Ni los políticos ni los medios de comunicación han contribuido a que ese sentimiento se moviese más cerca, psicológicamente, 'a Europa'. Las actitudes británicas vis-à-vis la UE fueron siempre distantes y reservadas, y peor aún, firmemente opuestas a todo lo que apareciese como una amenaza a la soberanía, identidad y costumbres nacionales [...]. (Álvarez, 2016, p. 29)

Los factores internos o domésticos de tipo coyuntural se relacionan con las situaciones de presión que recayeron sobre el primer ministro David Cameron. En primer lugar, el aumento del euroescepticismo dentro del Partido Conservador condujo a la propuesta de la consulta a la ciudadanía sobre su decisión de continuar o abandonar la Unión Europea; y en segundo lugar, el avance y la fortaleza que adquirió el United Kingdom Independence Party (UKIP), el cual surgió en la década de los 90 y una de sus banderas de creación 
fue lograr la salida del Reino Unido de la Unión (Álvarez, 2016; Virdee \& McGeever, 2018).

\section{Los resultados del referendo del 2016: ¿Qué motivó el voto?}

Tras los resultados del referendo, se llevaron a cabo diversos análisis con el objetivo de comprender e interpretar la posición, entre comillas, mayoritaria del pueblo de Gran Bretaña respecto del retiro del Estado de la Unión Europea. Lo primero que se debe señalar, pues resulta lo más evidente conforme a los datos, es que mientras la población del centro - Londres y cercanías- se oponía al Brexit y apoyaba el mantener el estatus de miembro de la Unión Europea, los ciudadanos de la periferia votaron por el abandono del bloque, excepto la población de Escocia e Irlanda del Norte:

Desde el punto de vista geográfico, de las nueve regiones que forman parte de Inglaterra, en ocho ganó el 'Leave'. La única excepción fue el Gran Londres, donde el 'Remain' se impuso con comodidad, por 60 a 40 por ciento. El 'Remain' se impuso categóricamente también en Irlanda del Norte, por 55,8 a 44,2 por ciento, y todavía más en Escocia, por 62 a 38 por ciento. (Álvarez, 2016, p. 30)

En parte, esto se debe a la percepción de desigualdad que tienen los miembros de la sociedad aislada respecto de las ciudades que albergan el poder financiero y económico. Para muchos, los resultados del referendo son una especie de rebelión de clase o una rebelión en contra del centro de poder, porque la Unión Europea ha representado solo beneficios para algunos sectores:

La sociedad británica, como otras europeas, cuenta con escasa movilidad social, de modo que las élites se caracterizan por la continuidad familiar mientras los menos favorecidos se mantienen en el lugar que tradicionalmente han ocupado. Si a esto añadimos que quienes han sufrido las medidas adoptadas para enfrentar la crisis económica han sido precisamente los indicados en último lugar, comprenderemos el motivo por el que los eslóganes de quienes hablan un lenguaje que entendemos y nos gusta escuchar suelen triunfar entre estos sectores sociales. (García \& García, 2017, p. 20)

Ahora bien, considerando la edad y la formación, se cuenta con datos que muestran que los ciudadanos más jóvenes y educados votaron por permanecer dentro de la Unión Europea, mientras que las personas mayores de los 35 y 40 años de clase trabajadora, pero desempleados o con empleos que no satisfacen sus pretensiones, votaron por el retiro de Gran Bretaña del bloque. Y precisamente este grupo, inconforme e insatisfecho, salió de manera masiva a las urnas. La percepción de estos sobre los resultados de la Unión Europea, es que esta solo ha beneficiado a los centros de poder y tan solo algunos sectores. 
Por lo anterior, Álvarez (2016) indica que los resultados del referendo fueron parejos, pero no homogéneos. La ubicación geográfica de los electores y la edad de los votantes, y otros factores sociodemográficos, permiten señalar que estos determinaron las pesquisas electorales del 2016. Por ello, se tuvo en cuenta una publicidad que hacia énfasis en temas como la migración, la seguridad, la cultura, la identidad, entre otros.

Se hicieron evidentes miedos, las fobias $\mathrm{y}$ las tendencias ultranacionalistas y xenofóbicas, producto de la libre circulación de migrantes y la idea de que estos representan una carga para los Estados (Virdee \& McGeever, 2018). Para muchos, el país se llenaría de millones de migrantes que afectarían la oferta laboral, la inversión social y la carga del Estado:

El argumento de que el Reino Unido no podía controlar el número de migrantes si permanecía como miembro de la UE fue una efectiva arma de campaña. Como también lo fueron los mitos tales como el pago de 350 millones de libras semanales de Reino Unido a Bruselas, o el inminente acceso de Turquía a la UE. (Álvarez, 2016, p. 30)

Claro está, para las empresas resulta esta situación favorable -la libertad migratoria-, porque la libre circulación de la mano de obra le permite mantener por debajo los salarios y contener el alza de estos, pero en el caso de los trabajadores nacionales esto implica menores ingresos y más dificultades para encontrar trabajo.

De acuerdo con García y García (2017), los resultados del referendo muestran de cierta manera una sociedad profundamente dividida, considerando que la participación de la ciudadanía fue la más alta desde 1980, el 72.2 \% de la ciudadanía participó en la consulta. Dentro de los motivos que llevaron a estos resultados se encuentran los efectos de las crisis económicas, el populismo y los recuerdos de otras épocas con este tipo de regímenes. Además, se encuentran el voto contra la oleada migratoria, los banqueros de Londres y las instituciones de la Unión Europea que llevan a un centralismo del poder en el continente.

Por último, algunos autores sugieren que la globalización tuvo influencia sobre el voto por el Brexit. La investigación de Colantone y Stanig (2018) sugiere que la angustia económica provocada por las importaciones chinas y las limitaciones de naturaleza laboral, condujeron a apoyar el "leave". En otras palabras, el neoliberalismo, que hace parte del fenómeno de la globalización, ha permitido el crecimiento económico y un mayor bienestar en grupos específicos de los países avanzados, pero los beneficios no han sido extensibles a todos por igual. De esta manera, hay grupos de individuos que se encuentran excluidos 
de las ventajas de la plena liberación económica, y esto, sumado a la incapacidad de los Estados para dar respuestas a las necesidades y exigencias sociales y económicas de los "abandonados de la globalización", puede explicar de cierta manera los resultados de las votaciones del Brexit en el 2016.

\section{LAS IMPLICACIONES GEOPO- LÍTICAS Y ECONÓMICAS DEL BREXIT}

Una búsqueda generalizada de fuentes a través de google scholar con las palabras claves "Brexit" e "implicaciones", muestra un total de 2100 resultados, y en inglés, la búsqueda de estos términos -Brexit + implications- genera más de 35000 resultados. Sin duda, esto muestra que se trata de uno de los principales temas de interés en la actualidad, pero, como bien afirma Gamble (2018), pasarán muchos años para lograr comprender las implicaciones y efectos del Brexit, y las votaciones de junio de 2016.

Antes de mostrar el análisis adelantado, parece prudente definir las categorías implicaciones geopolíticas e implicaciones económicas. La Real Academia Española indica que el término impli- cación proviene del latín implicatio y significa, en el contexto de este trabajo, "repercusión o consecuencia de algo". Ahora bien, debemos entender la geopolítica como aquellas interacciones y dinámicas que se trazan entre la geografía humana y física sobre la política y las relaciones internacionales; en otras palabras, el dominio político del espacio, el cual integra lo económico, lo social y lo cultural (Cuéllar, 2012). En relación con lo económico, interpretamos esta categoría como aquella que se asocia con la producción, la distribución y el consumo de bienes y servicios, y se expresa en variables económicas y macroeconómicas.

En las tablas 1 y 2 se describen algunas de las variadas implicaciones del Brexit localizadas dentro de la literatura. Cada una de estas es clasificada en alguna de las dos categorías previstas: geopolítica y económica. A su vez, cada implicación geopolítica y económica es sometida a un proceso de subcategorización para evidenciar de mejor forma los hallazgos obtenidos. Al final se muestra la fuente consultada que permitió el proceso de codificación y categorización. 
Tabla 1. Algunas implicaciones económicas del Brexit para Europa y Gran Bretaña

\begin{tabular}{|c|c|c|c|}
\hline Implicación & Clasificación & Subcategorización & Fuente \\
\hline \multirow{2}{*}{$\begin{array}{c}\text { Percepción negativa de las empresas } \\
\text { europeas (pesca marina) que comercializan } \\
\text { con empresas de Reino Unido }\end{array}$} & Económica & $\begin{array}{l}\text { Percepción } \\
\text { interempresas }\end{array}$ & Garza \& Amigo (2019) \\
\hline & Económica & Sectorial & Garza \& Amigo (2019) \\
\hline Afectación del turismo en Reino Unido & Económica & Sectorial & Castellote (2017) \\
\hline $\begin{array}{l}\text { Afectación de los derechos de propiedad } \\
\text { intelectual y propiedad industrial }\end{array}$ & Económica & $\begin{array}{l}\text { Propiedad intelectual } \\
\text { e industrial }\end{array}$ & De Miguel (2018) \\
\hline Afectación en el valor del precio del petróleo & Económica & Sectorial & Silveiro (2017) \\
\hline $\begin{array}{c}\text { Efectos sobre los impuestos de valor } \\
\text { añadido, derechos arancelarios o impuesto } \\
\text { de renta de personas físicas }\end{array}$ & Económica & Fiscal & Fondón (2017) \\
\hline Afectación de la libre circulación y la residencia & Económica & Laboral & Lozano (2017) \\
\hline Afectación de la libre circulación y la residencia & Económica & Migratoria & Lozano (2017) \\
\hline $\begin{array}{l}\text { Riesgos potenciales sobre la cadena de suministros } \\
\text { de las empresas al aumentar la complejidad } \\
\text { de los procesos aduaneros y otros aspectos }\end{array}$ & Económica & Cadena de suministro & Castillo (2017a) \\
\hline $\begin{array}{l}\text { Riesgos potenciales sobre la cadena de suministros } \\
\text { de las empresas al aumentar la complejidad } \\
\text { de los procesos aduaneros y otros aspectos }\end{array}$ & Económica & Procesos aduaneros & Castillo (2017a) \\
\hline Coordinación en los sistemas de seguridad social & Económica & Seguridad social & García (2018) \\
\hline $\begin{array}{l}\text { Pago a Reino Unido a la UE la suma de } \\
40000-45000 \text { millones de euros }\end{array}$ & Económica & Sanción & $\begin{array}{l}\text { Rodríguez \& } \\
\text { Pérez (2018) }\end{array}$ \\
\hline Depreciación de la libra esterlina & Económica & Monetaria & $\begin{array}{l}\text { Kierzenkowski } \\
\text { et al. (2016) } \\
\text { Hobolt (2016) }\end{array}$ \\
\hline $\begin{array}{l}\text { Disminución de los precios de los } \\
\text { activos financieros del país }\end{array}$ & Económica & Financiero & $\begin{array}{l}\text { Kierzenkowski, } \\
\text { et al. (2016) }\end{array}$ \\
\hline Pérdida masiva de empleos en Reino Unido & Económica & Laboral & $\begin{array}{l}\text { Rodríguez \& } \\
\text { Pérez (2018) }\end{array}$ \\
\hline $\begin{array}{l}\text { Disminución del crecimiento porcentual } \\
\text { de la UE con la salida de Reino Unido }\end{array}$ & Económica & $\begin{array}{l}\text { Crecimiento económico } \\
\text { en bloque }\end{array}$ & $\begin{array}{l}\text { Rodríguez \& } \\
\text { Pérez (2018) }\end{array}$ \\
\hline $\begin{array}{c}\text { Afectación de los negocios internacionales } \\
\text { y el mercado del Reino Unido / Creación } \\
\text { de barreras para el comercio }\end{array}$ & Económica & $\begin{array}{c}\text { Negocios } \\
\text { internacionales }\end{array}$ & $\begin{array}{l}\text { Cumming \& } \\
\text { Zahra (2016) }\end{array}$ \\
\hline $\begin{array}{c}\text { Afectación de políticas agrícolas y agroalimentaria } \\
\text { de la UE: regulación, presupuesto, } \\
\text { investigación y relaciones comerciales }\end{array}$ & Económica & Sectorial & Matthews (2016) \\
\hline $\begin{array}{l}\text { Nuevos acuerdos comerciales, por } \\
\text { ejemplo, un acuerdo de integración } \\
\text { comercial con China o EEUU }\end{array}$ & Económica & $\begin{array}{c}\text { Negocios } \\
\text { internacionales }\end{array}$ & $\begin{array}{c}\text { Colantone \& } \\
\text { Stanig (2018) } \\
\text { Brakman et al. (2018) }\end{array}$ \\
\hline $\begin{array}{l}\text { Reducción del PIB y decrecimiento } \\
\text { de la economía de Reino Unido }\end{array}$ & Económica & Macroeconómica & Born et al. (2017) \\
\hline $\begin{array}{l}\text { Disminución del intercambio comercial con } \\
\text { la UE, el principal socio del Reino Unido }\end{array}$ & Económica & $\begin{array}{l}\text { Crecimiento económico } \\
\text { en bloque }\end{array}$ & Sampson (2017) \\
\hline $\begin{array}{l}\text { Disminución en el crecimiento / } \\
\text { aumento de los salarios }\end{array}$ & Económica & Laboral & Sampson (2017) \\
\hline Aumento de la inflación & Económica & Microeconómico & Sampson (2017) \\
\hline $\begin{array}{l}\text { Decrecimiento en el ritmo de } \\
\text { producción del Reino Unido }\end{array}$ & Económica & Microeconómico & Sampson (2017) \\
\hline $\begin{array}{l}\text { Nuevas regulaciones en cuanto al } \\
\text { sistema energético: impacto menor }\end{array}$ & Económica & Energía & Pollitt (2017) \\
\hline
\end{tabular}


Tabla 2. Algunas implicaciones geopolíticas del Brexit para Europa y Gran Bretaña

\begin{tabular}{|c|c|c|c|}
\hline Implicación & Clasificación & Sub categorización & Fuente \\
\hline Fractura socioterritorial en Escocia & Geopolítica & Social & García \& García (2017) \\
\hline Fractura de partidos políticos tradicionales & Geopolítica & Política & García \& García (2017) \\
\hline $\begin{array}{l}\text { Pérdida de uno de los ejércitos más } \\
\text { grandes por parte de la UE }\end{array}$ & Geopolítica & Seguridad y defensa & $\begin{array}{l}\text { Riordan (2016) } \\
\text { Pontijas (2018) } \\
\text { Rabanal (2018) }\end{array}$ \\
\hline $\begin{array}{c}\text { Aumento de riesgos a amenazas externas, } \\
\text { v.g. terrorismo. UE y Reino Unido }\end{array}$ & Geopolítica & Seguridad y defensa & Pontijas (2018) \\
\hline $\begin{array}{l}\text { Mejora de las capacidades de la UE } \\
\text { en materia de seguridad y defensa }\end{array}$ & Geopolítica & Seguridad y defensa & $\begin{array}{c}\text { Pontijas (2018) } \\
\text { Weyembergh (2017) }\end{array}$ \\
\hline $\begin{array}{l}\text { Reino Unido pierde su papel como } \\
\text { portavoz de Washington en Europa }\end{array}$ & Geopolítica & Relaciones internacionales & Riordan (2016) \\
\hline $\begin{array}{c}\text { Aumento del gasto en defensa } \\
\text { para Reino Unido }\end{array}$ & Geopolítica & Fiscal & Riordan (2016) \\
\hline $\begin{array}{l}\text { Inicio de la fragmentación de } \\
\text { la UE o desmantelamiento del } \\
\text { bloque como se conoce hoy }\end{array}$ & Geopolítica & Divisiones dentro de la UE & Castillo (2017b) \\
\hline $\begin{array}{l}\text { Incertidumbres sobre los derechos } \\
\text { de los ciudadanos con residencia, } \\
\text { permanencia y trabajo en Reino Unido }\end{array}$ & Geopolítica & Social, jurídico DDHH & Kilkey (2017) \\
\hline $\begin{array}{l}\text { Afectación de las "familias } \\
\text { Brexit" y sus derechos }\end{array}$ & Geopolítica & Social, jurídico DDHH & Kilkey (2017) \\
\hline $\begin{array}{l}\text { Afectación de la gobernanza y la } \\
\text { economía política del Reino Unido }\end{array}$ & Geopolítica & Política, democracia & Gamble (2018) \\
\hline $\begin{array}{l}\text { Sumisión abyecta a la administración } \\
\text { derechista más errática de los EEUU }\end{array}$ & Geopolítica & $\begin{array}{l}\text { Política, relaciones } \\
\text { internacionales }\end{array}$ & $\begin{array}{c}\text { Gumbrell \& } \\
\text { Hyman (2017) }\end{array}$ \\
\hline $\begin{array}{c}\text { Derechos laborales en general y } \\
\text { participación sindical en Reino Unido }\end{array}$ & Geopolítica & $\begin{array}{l}\text { Social, jurídico } \\
\text { DDHH, laboral }\end{array}$ & $\begin{array}{c}\text { Gumbrell \& } \\
\text { Hyman (2017) }\end{array}$ \\
\hline $\begin{array}{l}\text { Limitaciones en el intercambio de } \\
\text { información con las autoridades policiales, } \\
\text { por ejemplo, con Noruega o Suiza }\end{array}$ & Geopolítica & Seguridad y defensa & $\begin{array}{c}\text { Josifovic \& Keskoski } \\
\text { (2017) }\end{array}$ \\
\hline $\begin{array}{l}\text { Limitaciones para la consulta directa e } \\
\text { independiente de información, v.g. Europol }\end{array}$ & Geopolítica & Información / Redes & $\begin{array}{c}\text { Josifovic \& } \\
\text { Keskoski (2017) }\end{array}$ \\
\hline $\begin{array}{l}\text { Reducción de funcionarios de Reino } \\
\text { Unido y que ocupan cargos en } \\
\text { entidades o instituciones de la UE }\end{array}$ & Geopolítica & $\begin{array}{l}\text { Participación en el campo } \\
\text { operativo en agencias } \\
\text { y entidades de la UE }\end{array}$ & $\begin{array}{c}\text { Josifovic \& } \\
\text { Keskoski (2017) }\end{array}$ \\
\hline $\begin{array}{l}\text { Afectación de la igualdad de género en } \\
\text { el proceso de negociación: incertidumbre } \\
\text { sobre la participación de la mujer }\end{array}$ & Geopolítica & Participación / género & $\begin{array}{c}\text { Guerrina \& } \\
\text { Masselot, (2018) }\end{array}$ \\
\hline
\end{tabular}

El análisis de estos datos de las tablas 1 y 2 se puede visualizar en las figuras de los anexos, las cuales se refieren a las redes semánticas sobre impacto económico y geopolítico, respectivamente. Valga resaltar que estas categorías surgieron de un proceso de codificación en donde las unidades textuales sir- vieron de base para la construcción de códigos según el sentido allí implícito. De esa manera, las categorías no fueron seleccionadas a priori, sino que emergen del proceso analítico que implica la codificación y categorización de las unidades de análisis. 
El principal socio comercial del Reino Unido ha sido la Unión Europea. Sus exportaciones e importaciones en relación con el PIB han sido del 0,57 con la Unión Europea, y tan solo del 0,28 con los Estados Unidos (Sampson, 2017). En términos de exportaciones, la Unión Europea ha sido el destino del $44 \%$ de los bienes y servicios del Reino Unido, y las importaciones de la Unión Europea han correspondido al $53 \%$ del total. Sin duda, el principal socio comercial del Reino Unido en las últimas décadas ha sido la Unión Europea y, por tanto, la importancia de este mercado para el Reino Unido es significativa e invaluable.

El intercambio económico y la integración comercial del Reino Unido frente a la Unión Europea, su socio principal, corresponde a uno de los principales temas de interés para los analistas y expertos. Por ello, muchos de los estudios, investigaciones y análisis realizados en los últimos años frente al Brexit, se han centrado de manera particular en este punto. Sin embargo, los casos previos de Argelia (1962) o Groelandia (1985) no pueden brindar mucha luz sobre las consecuencias económicas que traerá el Brexit tanto para el Reino Unido como para la Unión Europea. Pese a lo anterior, los estudios al respecto han introducido tres tipos de enfoques con el objetivo de precisar los impactos económicos: 1. Estudios de casos en la historia y las consecuencias de estos de unirse a la Unión Europea -por analogía-, 2. Simulaciones de Brexit utilizando modelos comerciales de equilibrio computacional, y 3. Estimaciones a partir de la forma en que afecta el comercio la pertenencia a la Unión Europea (Sampson, 2017).

Dentro del ámbito económico, y considerando los primeros efectos tras el referendo del 2016, los principales impactos inmediatos del Brexit para el Reino Unido son: aumento gradual de la inflación, desaceleración de la producción, depreciación de la moneda frente al dólar y una baja en el aumento porcentual de los salarios (Sampson, 2017). Se trata, claro está, de efectos inmediatos que se agudizarán o modificarán en favor del Reino Unido según como se marquen las negociaciones para su salida.

En el estudio de Brakman et al. (2018) se expresa que tras la salida del Reino Unido del bloque, se hace posible un acuerdo comercial bilateral con Estados Unidos. Por otro lado, Colantone y Stanig (2018) indican que es posible que el Reino Unido se acelere en confeccionar y cerrar un trato comercial con China. En todo caso, ambos deberán sopesar las pérdidas que resulten de abandonar la Unión Europea, principal socio comercial del Reino Unido.

Para Brakman et al. (2018), un acuerdo comercial con Estados Unidos puede aumentar la exportación de valor agregado para ambos países (entre $2 \%$ y $4 \%$ ), y con ello compensar de alguna manera el impacto del Brexit. Y un 
acuerdo comercial con China podría tener efectos similares, pero sin que se compensen plenamente las pérdidas por el Brexit. Desde esta perspectiva, vale la pena que la salida del Reino Unido de la Unión Europea se haga de manera negociada, de tal forma que todas las alternativas para un intercambio comercial -anteriores con miembros de la Unión Europea y nuevas, por ejemplo, Estados Unidos o China- impliquen beneficios compensatorios.

De igual manera, en el esquema económico, el Brexit puede generar otros impactos para el Reino Unido y la Unión Europea. Por ejemplo, en un escenario práctico, algunas empresas pueden abandonar las relaciones y el intercambio mercantil con el Reino Unido, tal es el caso de las empresas del sector pesquero (Garza \& Amigo, 2019). También se suman algunas afectaciones o riesgos en sectores específicos de la economía: el turismo (Castellote, 2017), el precio del petróleo (Silveiro, 2017), la cadena de suministro de las empresas (Castillo, 2017a), sector agrícola y seguridad alimentaria (Matthews, 2016), energía (Pollitt, 2017), entre otros.

Otros efectos también de orden económico del Brexit, pero relacionados con el tema laboral, que afectan a los trabajadores, son: libre circulación y residencia de los trabajadores de parte y parte (Lozano, 2017), dificultades en el manejo coordinado del sistema de seguridad social de los trabajadores (García, 2018), pérdida de puestos de trabajo en el Reino Unido (Rodríguez \& Pérez, 2018), afectación de derechos laborales en general y de la participación sindical en Reino Unido (Gumbrell \& Hyman, 2017), y otros.

Ahora bien, en el terreno de la geopolítica se describen variados posibles efectos del Brexit para los actores implicados. Dentro de los temas de mayor interés expuestos en la literatura son: pérdida de ejércitos para la Unión Europea (Riordan, 2016; Pontijas, 2018; Rabanal, 2018), aumento de riesgos y amenazas terroristas (Pontijas, 2018), pérdida del papel de portavoz del Reino Unido frente a Washington (Riordan, 2016), incertidumbre respecto de los derechos de los ciudadanos con residencia en Reino Unido o las "familias Brexit"2 (Kilkey, 2017), afectación de los procesos de gobernanza (Gamble, 2018), sumisión del Reino Unido a la derecha más errática de los Estados Unidos (Gumbrell \& Hyman, 2017), entre otras.

Por último, no se pueden dejar de lado las implicaciones que podría traer el Covid-19 sobre la economía del Reino Unido, aunque sobre esto no hay investigaciones aún, siendo una línea de estudio interesante para estos tiempos de crisis de la Unión Europea. En efecto, el Reino Unido parece quedarse aislado

2 Las "familias Brexit" se refieren al conjunto de grupos familiares migrantes, bien de la Unión Europea dentro del Reino Unido, o bien, familias del Reino Unido que se encuentran en otros países de la Unión Europea y que, gracias al Brexit, se posibilita la migración y el desarrollo de proyectos de vida en común. 
en un momento en que se requieren mayores esfuerzos de cooperación e integración regional. Por otro lado, la emergencia sanitaria ha obligado a que se aplacen las rondas de negociaciones, las cuales permiten determinar las reglas hacia el futuro en materia de relaciones económicas del Reino Unido con la Unión Europea. Se puede prever que la crisis que vive hoy la Unión Europea en su economía y sistema de salud puede extenderse en igual magnitud al Reino Unido, pero este último no tendrá las ayudas económicas que cobijarán a los otros miembros de la Unión Europea ${ }^{3}$.

\section{CONCLUSIONES}

El Brexit configura un fenómeno complejo nada fácil de comprender e interpretarse, pero, aun así, se trata de un caso que no tiene precedente en el campo de las relaciones internacionales, la política y el comercio exterior. $\mathrm{Su}$ análisis puede llevar a una revisión de aspectos relevantes asociados con el tema de la integración y, particularmente, sobre el futuro de la Unión Europea. De acuerdo con los hallazgos obtenidos, las implicaciones económicas $\mathrm{y}$ geopolíticas del Brexit son múltiples, aunque destacan dentro de la literatura aquellos efectos relacionados con el comercio internacional, los derechos de los trabajadores y las familias Brexit, la contracción de determinados sectores económicos y actividades comerciales, y la configuración de nuevas alianzas y posturas en lo económico y geopolítico.
El Brexit, sin duda, es el comienzo de un proceso de reconfiguración de la Unión Europea, que deberá modificarse de manera flexible para adaptarse a las necesidades y expectativas de los países miembros, so pena de generar más retiros en el futuro. El caso analizado pone de manifiesto la necesidad de revisar el fenómeno de la integración en pleno siglo XXI y la urgencia de verificar los resultados positivos de la integración entre los Estados y las sociedades. Además, corresponde a un hecho en el que es fundamental considerar las tendencias ultranacionalistas y xenofóbicas, las cuales son cada vez más marcadas y extendidas.

Por último, se debe señalar que pese a las variadas posibles consecuencias y efectos del Brexit, aún resulta imposible identificar con cierto grado de certeza los resultados de la salida del Reino Unido de la Unión Europea. Como señalan muchos autores, esto dependerá del tiempo y de las maniobras que haga el Reino Unido en términos de negociación con la Unión Europea y otros aliados comerciales desde el mismo momento del retiro.

\section{DECLARACIÓN DE CONFLICTOS DE INTERÉS}

Los autores declaran que no existe conflicto de intereses

3 El 10 de marzo se aprobó un fondo de respuesta de 25000 millones de euros para atender la emergencia sanitaria. 


\section{REFERENCIAS}

Altmann, J. (2011). Entre la unión y la desunión: alcances y limitaciones de la integración centroamericana. IdeAs. https://doi.org/10.4000/ideas.85

Álvarez, M. (2012). El euroescepticismo en una Unión Europea en crisis: ¿viejo fenómeno en nuevos odres? Revista Integración y Cooperación Internacional, (13), 3-17. http://rephip.unr.edu.ar/handle/2133/5684

Álvarez, M. (2016). La salida del Reino Unido de la Unión Europea: sus causas estructurales. Revista Cooperación e Integración Internacional, (23), 27-31. https://core.ac.uk/download/pdf/61707807.pdf

Ánima, S. (2017). La Unión Europea y su problemática de integración. Economía Informa, 403(2), 4-20.

Belke, A., Dubova, I., \& Osowski, T. (2018). Policy Uncertainty and International Financial Markets: The Case of Brexit. Applied Economics, 50(34-35), 37523770. https://doi.org/10.1080/00036846.2018.1436152

Bonilla, E. \& Rodríguez, P. (2005). Más allá del dilema de los métodos: la investigación en ciencias sociales. Norma.

Born, B., Müller, G., Schularick, M. \& Sedláček, P. (2017). The Economic Consequences of the Brexit Vote. CESifo Working Paper, No. 6780. Center for Economic Studies and Ifo Institute (CESifo).

Brakman, S., Garretsen, H. \& Kohl, T. (2018). Consequences of Brexit and Options for a 'Global Britain'. Regional Science, 97(1), 55-72. https://doi.org/10.1111/ pirs. 12343

Caporaso, J. (2018). The European Union: Dilemmas of Regional Integration. Routledge.

Castellote, Y. (2017). Brexit: implicaciones del fenómeno político social en el contexto británico y su turismo. (Tesis de grado). Universidad de Valladolid, Valladolid, España. http://uvadoc.uva.es/handle/10324/24058

Castillo, J. (2017a). El Brexit: causas, consecuencias y alternativas futuras. (Tesis de grado). Universidad de Sevilla, Sevilla, España. 
Castillo, F. (2017b). Impacto del brexit en las empresas del Reino Unido y sus sucursales en la UE. (Tesis de grado). Universitat Oberta de Catalunya, Catalunya, España. http://openaccess.uoc.edu/webapps/o2/handle/10609/74166

Compains, E. (2017). El impacto del Brexit sobre el estatus jurídico-político de Irlanda del Norte: ¿Una oportunidad para la reunificación de Irlanda? Revista Vasca de Administración Pública, (109), 59-98.

Colantone, I. \& Stanig, P. (2018). Global Competition and Brexit. American Political Science Review, 112(2), 201-218. https://doi.org/10.1017/S0003055417000685

Cuéllar, R. (2012). Geopolítica. Origen del concepto y su evolución. Revista de Relaciones Internacionales de la UNAM, (113), 59-80.

Cumming, D. \& Zahra, S. (2016). International Business and Entrepreneurship Implications of Brexit. British Journal of Management, 27(4), 687-692.

Daddow, O. (2015). Interpreting the Outsider Tradition in British European Policy Speeches from Thatcher to Cameron. Journal of Common Market Studies, 53(1), 71-88. https://doi.org/10.1111/jcms.12204

Debomy, D. (2012). Do the Europeans Still Believe in the EU? Analysis of Attitudes and Expectations of EU Public Opinions over the Past Quarter Century. Study $\&$ Research, (91). https://www.europeansources.info/record/do-the-europeansstill-believe-in-the-eu-analysis-of-attitudes-and-expectations-of-eu-publicopinions-over-the-past-quarter-century/

De Miguel, P. (2018). Implicaciones del Brexit sobre los derechos de propiedad industrial e intelectual: estado de la cuestión. La Ley Unión Europea, (60).

Dosenrode, S. (2016). Limits to Regional Integration. Routledge.

Fondón, A. (2017). Posibles efectos tributarios del Brexit. Anales: Anuario del Centro de la UNED de Calatayud, (23), 91-107.

Franco, A. \& Robles, F. (1995). Integración: un marco teórico. Colombia Internacional, (30), 16-22.

Gamble, A. (2018). Taking Back Control: The Political Implications of Brexit. Journal of European Public Policy, 25(8), 1215-1232. 
García, C. (2018). El Brexit en el ámbito de la coordinación de regímenes de seguridad social. Revista General de Derecho del Trabajo y de la Seguridad Social, (49).

García, S. \& García, P. (2017). ¿Qué es el Brexit? Origen y posibles consecuencias. Anuario Mexicano de Derecho Internacional, 17, 3-40.

Garza, M. \& Amigo, L. (2019). La política pesquera común y el brexit. Implicaciones para las empresas españolas. Estudios de Economía Aplicada, 37(3), 113-132.

Graziano, A., Handley, K. \& Limão, N. (2018) Brexit Uncertainty and Trade Disintegration. NBER Working Paper, (25334), 1-49. https://www.nber.org/ papers/w25334.pdf

Guerrina, R. \& Masselot, A. (2018). Walking into the Footprint of EU Law: Unpacking the Gendered Consequences of Brexit. Social Policy and Society, 17(2), 319-330. https://doi.org/10.1017/S1474746417000501

Guinea, J. (2018). El proceso de consolidación del euroescepticismo británico y el triunfo del Brexit: fuerzas centrípetas para el reforzamiento de la política exterior y la política de defensa de la Unión Europea. Cadernos de Dereito Actual, (9), 305-331. https://dialnet.unirioja.es/servlet/articulo?codigo=6484835

Gumbrell, R. \& Hyman, R. (2017). What about the Workers? The Implications of Brexit for British and European Labour. Competition \& Change, 21(3), 169184. https://doi.org/10.1177/1024529417698514

Hernández, R., Fernández, C. \& Baptista, P. (2014). Metodología de la investigación. McGraw Hill.

Hobolt, S. (2016). The Brexit Vote: A Divided Nation, a Divided Continent. Journal of European Public Policy, 23(9), 1259-1277. https://doi.org/10.1080/13501763. 2016.1225785

Josifovic, I. \& Keskoski, Z. (2017). European Union's Area of Freedom, Security and Justice after Brexit: Consequences and Future Prospects. Vizione, (28), 425-433. http://eprints.ugd.edu.mk/19379/

Kierzenkowski, R., Pain, N., Rusticelli, E. \& Zwart, S. (2016). The Economic Consequences of Brexit: A Taxing Decision. OECD Publishing. 
Kilkey, M. (2017). Conditioning Family-Life at the Intersection of Migration and Welfare: The Implications for 'Brexit Families'. Journal of Social Policy, 46(4), 797-814.

Lanchester, J. (2016). Brexit Blues. Letras Libres, (180), 4-10. https://www.letraslibres.com/sites/default/files/2016-09/Dosier-lanchester-esp.pdf

Laursen,F.(2016). Comparative Regional Integration. EuropeandBeyond. Routledge.

Lozano, B. (2017). Las implicaciones del Brexit en los derechos de libre circulación de personas, laborales y de residencia de los ciudadanos de la Unión Europea: el modelo de Suiza modificado como la opción más viable para el Reino Unido en la negociación de un nuevo acuerdo con la Unión Europea. (Tesis de grado). Instituto Tecnológico y de Estudios Superiores de Monterrey, Monterrey, México.

Matthews, A. (2016). The Potential Implications of a Brexit for Future EU Agri-Food Policies. EuroChoices, 15(2), 17-23.

Mogalakwe, M. (2006). The Use of Documentary Research Methods in Social Research. African Sociological Review, 10(1), 221-230. https://doi.org/10.1353/ eas. 0.0006

Paniagua, J. (2018). Antecedentes, consecuencias y paradojas del Brexit. Razón y $\mathrm{Fe}, 277(1431), 87-97$. https://revistas.comillas.edu/index.php/razonyfe/article/ view/9092

Pérez, C. (2016). El Brexit y sus implicaciones jurídico-laborales en los desplazamientos de trabajadores de la Unión Europea. Actualidad Jurídica Arazandi, (921), 6.

Pollitt, M. (2017). The Economic Consequences of Brexit: Energy. Oxford Review of Economic Policy, 33(1), S134-S143. https://doi.org/10.1093/oxrep/grx013

Pontijas, J. (2018). Implicaciones del Brexit en los campos de la defensa y la seguridad. Boletín IEEE, (9), 651-668.

Rabanal, N. (2018). 'Brexit' y política de seguridad y defensaposibles efectos en las relaciones UE-UK. Boletín IEEE, (12), 858-885.

Riordan, J. (2016). 'Brexit': implicaciones para la seguridad europea. Documento de trabajo. Instituto Español de Estudios Estratégicos. 
Rodríguez, L. \& Pérez, E. (2018). El Brexit en las relaciones Reino Unido-Unión Europea. Impactos geopolíticos. Revista CCCS, (7). https://www.eumed.net/ rev/cccss/2018/07/brexit-impactos-geopoliticos.html

Sampson, T. (2017). Brexit: The Economics of International Disintegration. The Journal of Economic Perspectives, 31(4), 163-184. https://doi.org/10.1257/ jep.31.4.163

Signorelli, S. (2012). The EU and Public Opinions: A Love-Hate Relationship? Notre Europe, Jacques Delors Institute.

Silveiro, Y. (2017). El Brexit, la UE y las incertidumbres estratégicas: implicaciones a corto, mediano y largo plazo para África Subsahariana. Revista Brasileira de Estudos Africanos, 2(3), 10-37.

Strauss, A. \& Corbin, J. (2004). Bases de la investigación cualitativa. Técnicas yprocedimientos para desarrollar la teoría fundamentada. Universidad de Antioquia.

Steinberg, J. (2019). Brexit and the Macroeconomic Impact of Trade Policy Uncertainty. Journal of International Economics, 117, 175-195. https://oi. org/10.1016/j.jinteco.2019.01.009

Tokatlian, J. (1994). Componentes políticos de la integración. En J. Acosta (comp.), Integración, desarrollo económico y competitividad. Centro Regional de Estudios del Tercer Mundo, CRE-SET.

Virdee, S. \& McGeever, B. (2018). Racism, Crisis, Brexit. Ethnic and Racial Studies, 41(10), 1802-1819. https://doi.org/10.1080/01419870.2017.1361544

Weyembergh, A. (2017). Consequences of Brexit for European Union Criminal Law. New Journal of European Criminal Law, 8(3), 284-299. https://doi. org/10.1177/2032284417721286 


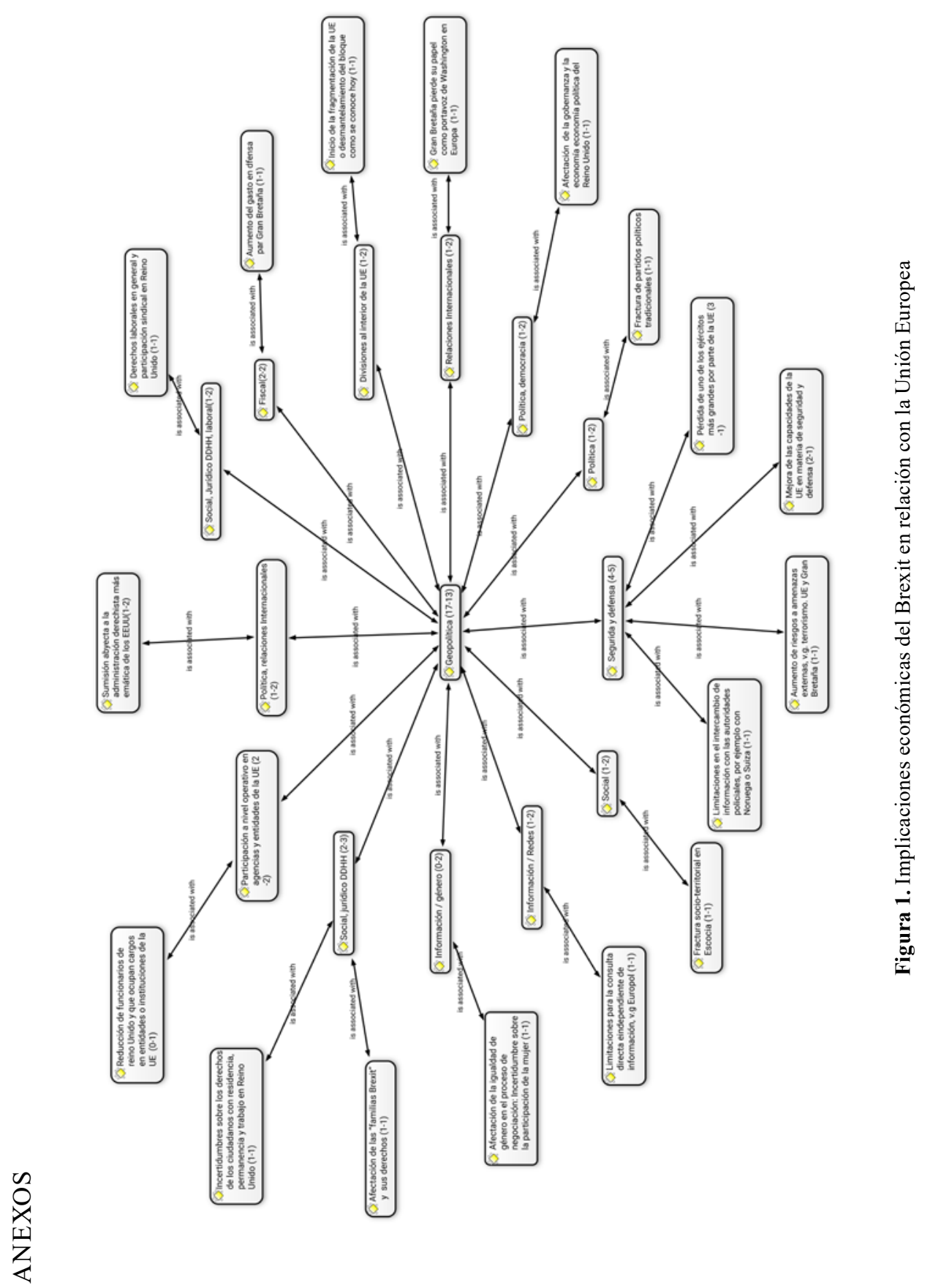




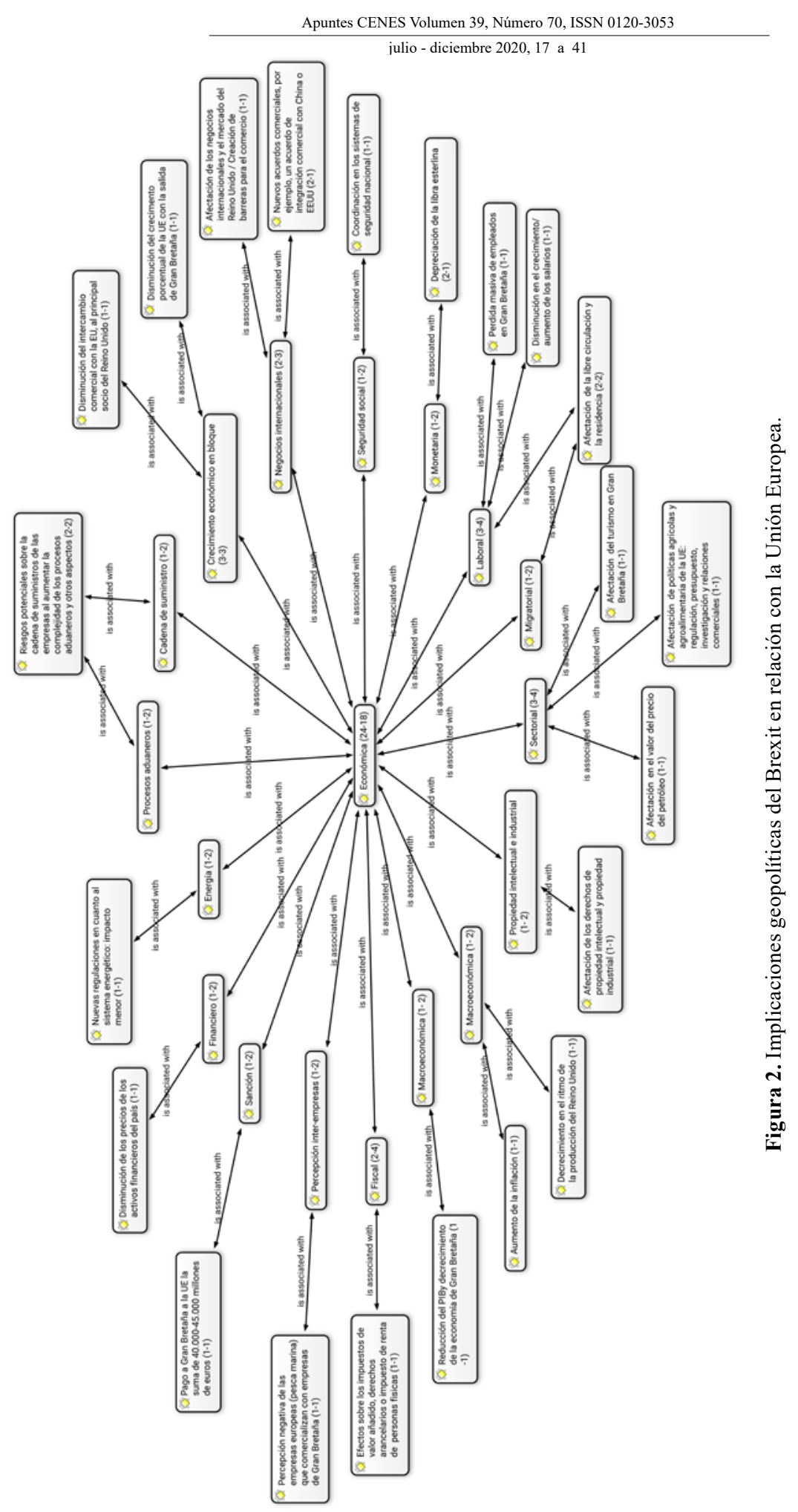

